\title{
A NEW CHARACTERISTIC PROPERTY OF MINIMAL SURFACES
}

\author{
EDWARD KASNER AND JOHN DECICCO
}

1. Isothermal families on a surface $\Sigma$ defined by the Monge equation $z=f(x, y)$. We shall present a new characteristic property of minimal surfaces (Theorem 3 ). We also discover an extensive class of surfaces (Theorem 1), including minimal surfaces and surfaces of revolution. ${ }^{1}$ It is shown that the minimal surfaces are the only ones such that every set of parallel planes intersects the surface in an isothermal family (except for the obvious case of a sphere).

In our preceding work, ${ }^{2}$ we have obtained an extension of the theorem of Lie concerning isothermal families in the plane. We have found that a family of curves: $g(x, y)=$ const., on a surface $\Sigma$ where any point is defined by general curvilinear coordinates $(x, y)$, is isothermal if and only if the angle $\theta=\theta(x, y)$ between the family and the parametric curves $x=$ const. satisfies a certain partial differential equation of the second order in $(x, y)$, and therefore if and only if $g(x, y)$ satisfies a certain partial differential equation of the third order in $(x, y)$. Of course, if $(x, y)$ are isothermal parameters on $\Sigma$, then our condition reduces to Lie's theorem which states that $\theta$ is a harmonic function of $(x, y)$ if and only if the given family of curves is isothermal.

We have applied our result to the case in which the surface $\Sigma$ is given by the Monge equation: $z=f(x, y)$, where $(x, y, z)$ denote cartesian coordinates of space. The condition is

$$
\begin{aligned}
\left(1+f_{y}^{2}\right) \frac{\partial^{2} \theta}{\partial x^{2}} & -2 f_{x} f_{y} \frac{\partial^{2} \theta}{\partial x \partial y}+\left(1+f_{x}^{2}\right) \frac{\partial^{2} \theta}{\partial y^{2}} \\
& -\left(1+f_{x}^{2}+f_{y}^{2}\right)^{-1}\left[\left(1+f_{y}^{2}\right) f_{x x}-2 f_{x} f_{y} f_{x y}\right. \\
& \left.+\left(1+f_{x}^{2}\right) f_{y y}\right]\left[f_{x} \frac{\partial \theta}{\partial x}+f_{y} \frac{\partial \theta}{\partial y}\right]+\left(1+f_{x}^{2}+f_{y}^{2}\right)^{1 / 2} \\
& \cdot \frac{\partial}{\partial y}\left\{\frac{f_{x}\left[\left(1+f_{y}^{2}\right) f_{x x}-2 f_{x} f_{y} f_{x y}+\left(1+f_{x}^{2}\right) f_{y y}\right]}{\left(1+f_{y}^{2}\right)\left(1+f_{x}^{2}+f_{y}^{2}\right)}\right\}=0,
\end{aligned}
$$

Presented to the Society, February 24, 1945; received by the editors March 2, 1945, and, in revised form, May 14, 1945.

1 The work of the present paper is concerned not only with real euclidean space but also with the complex euclidean space.

${ }^{2}$ Kasner and DeCicco, An extension of Lie's theorem on isothermal families, Proc. Nat. Acad. Sci. U.S.A. vol. 31 (1945) pp. 44-50. 
where $\theta=\theta(x, y)$ is the angle between the family of curves defined by the differential equation $d y / d x=p=p(x, y)$ and the parallel plane sections $x=$ const. The angle $\theta$ is given by the formula

$$
\theta=\operatorname{arccot} \frac{p\left(1+f_{y}^{2}\right)+f_{x} f_{y}}{\left(1+f_{x}^{2}+f_{y}^{2}\right)^{1 / 2}} .
$$

The two preceding formulas possess some symmetry but not total symmetry since the surface $\Sigma$ is defined by $z=f(x, y)$ and $\theta$ is the inclination to the parallel plane sections $x=$ const. These formulas may be put into totally symmetric form by letting $\theta$ represent the angle between the given family of curves defined by the differential equation of first order $d y / d x=p=p(x, y)$ and the parallel plane sections $z=$ const. Then formula (1) assumes the form

$$
\begin{aligned}
\left(1+f_{y}^{2}\right) \frac{\partial^{2} \theta}{\partial x^{2}}-2 f_{x} f_{y} \frac{\partial^{2} \theta}{\partial x \partial y}+\left(1+f_{x}^{2}\right) \frac{\partial^{2} \theta}{\partial y^{2}} \\
-\left(1+f_{x}^{2}+f_{y}^{2}\right)^{-1}\left[\left(1+f_{y}^{2}\right) f_{x x}-2 f_{x} f_{y} f_{x y}+\left(1+f_{x}^{2}\right) f_{y y}\right] \\
\cdot\left[f_{x} \frac{\partial \theta}{\partial x}+f_{y} \frac{\partial \theta}{\partial y}\right]+\left(1+f_{x}^{2}+f_{y}^{2}\right)^{1 / 2}\left[f_{y} \frac{\partial}{\partial x}-f_{x} \frac{\partial}{\partial y}\right] \\
\cdot\left\{\frac{\left(1+f_{y}^{2}\right) f_{x x}-2 f_{x} f_{y} f_{x y}+\left(1+f_{x}^{2}\right) f_{y y}}{\left(f_{x}^{2}+f_{y}^{2}\right)\left(1+f_{x}^{2}+f_{y}^{2}\right)}\right\}=0 .
\end{aligned}
$$

The angle $\theta$ is given by the expression

$$
\theta=\arctan \frac{\left(f_{x}+p f_{y}\right)\left(1+f_{x}^{2}+f_{y}^{2}\right)^{1 / 2}}{f_{y}-p f_{x}} .
$$

2. Application to minimal surfaces and spheres. Minimal surfaces may be defined as those for which the mean curvature is identically zero. Thus the surface $\Sigma$ defined by the Monge equation, $z=f(x, y)$, is minimal if and only if $f$ satisfies the partial differential equation of the second order

$$
\left(1+f_{y}^{2}\right) f_{x x}-2 f_{x} f_{y} f_{x y}+\left(1+f_{x}^{2}\right) f_{y y}=0 .
$$

By means of (1) or (3), and (5), it follows that a family of curves on the minimal surface $\Sigma$ is isothermal if and only if the angle $\theta$ between the given family and any one of the parallel plane sections $x=$ const., $y=$ const., or $z=$ const. satisfies the partial differential equation of second order 


$$
\left(1+f_{y}^{2}\right) \frac{\partial^{2} \theta}{\partial x^{2}}-2 f_{x} f_{y} \frac{\partial^{2} \theta}{\partial x \partial y}+\left(1+f_{x}^{2}\right) \frac{\partial^{2} \theta}{\partial y^{2}}=0 .
$$

If the condition (1) or (3) is independent of the first order partial derivatives of $\theta$, then $\Sigma$ must be a minimal surface. If in addition the coefficients of the partial derivatives of $\theta$ in (6) are proportional to real constants, then $\Sigma$ is a plane. For $\theta$ to be a harmonic function of $(x, y)$, we find that $\Sigma$ must be a plane parallel to the $(x, y)$-plane.

Let us apply the result (3) to the sphere $x^{2}+y^{2}+z^{2}=r^{2}$. A family of curves defined by the differential equation $d y / d x=p=p(x, y)$ on this sphere is isothermal if and only if the angle $\theta$ between the family and the circles of latitude $z=$ const. satisfies the partial differential equation of the second order

(7) $\left(r^{2}-x^{2}\right) \frac{\partial^{2} \theta}{\partial x^{2}}-2 x y \frac{\partial^{2} \theta}{\partial x \partial y}+\left(r^{2}-y^{2}\right) \frac{\partial^{2} \theta}{\partial y^{2}}-2\left(x \frac{\partial \theta}{\partial x}+y \frac{\partial \theta}{\partial y}\right)=0$.

The angle $\theta$ is given by

$$
\theta=\arctan \frac{r(x+p y)}{(y-p x)\left(r^{2}-x^{2}-y^{2}\right)^{1 / 2}} .
$$

If $\Sigma$ is a minimal surface or a sphere, then every system of parallel plane sections is isothermal.

3. Surfaces with an isothermal system of parallel plane sections. By equation (3), it is observed that the system of parallel plane sections $z=$ const. is isothermal if and only if

$$
\frac{\left(1+f_{y}^{2}\right) f_{x x}-2 f_{x} f_{y} f_{x y}+\left(1+f_{x}^{2}\right) f_{y y}}{\left(f_{x}^{2}+f_{y}^{2}\right)\left(1+f_{x}^{2}+f_{y}^{2}\right)}=\phi(f) .
$$

Let $K_{m}$ denote the mean curvature of the surface $\Sigma$ and let $\gamma$ denote the angle between the normal of $\Sigma$ and the common normal of any system of parallel plane sections. From (9), we deduce the following:

THEOREM 1. A given system of parallel plane sections of a surface $\Sigma$ is isothermal if and only if the product $K_{m} \cot \gamma \csc \gamma$ is the same at all points of any one of the plane sections.

Upon the plane sections of Theorem 1 we impose the additional condition that $K_{m}$ be the same at all points of any one of these sections. In the first place $K_{m}$ may be identically zero, in which case $\Sigma$ must be a minimal surface. Next let $K_{m} \neq 0$. Then $\gamma$ must be the same at all points of any one of these plane sections. Upon letting these 
plane sections be $z=$ const., then $\gamma=\gamma(f)$. Thus $\tan ^{2} \gamma=f_{x}^{2}+f_{y}^{2}=\psi(f)$. We find from this that

$$
f_{x} f_{x x}+f_{y} f_{x y}=\psi_{f} f_{x} / 2, \quad f_{x} f_{x y}+f_{y} f_{y y}=\psi_{f} f_{y} / 2
$$

Multiplying these by $f_{x}$ and $f_{y}$ respectively and adding the resulting products to the equivalent form of equation (9), obtained by clearing of fractions, we find

$$
(1+\psi)\left(f_{x x}+f_{y y}\right)=\phi \psi(1+\psi)+\psi \psi_{f} / 2 .
$$

From this it follows that $\left(f_{x x}+f_{y y}\right)$ must be a function of $f$ alone since $\psi \neq-1$. Since $f_{x}^{2}+f_{y}^{2}=\psi(f)$, these results mean that the orthogonal projections of the parallel plane sections upon the $(x, y)$-plane must be a parallel and an isothermal family. These projections are then either concentric circles or parallel straight lines. From this we deduce that $\Sigma$ must be either a surface of revolution with axis perpendicular to the $(x, y)$-plane or a cylinder with elements parallel to the $(x, y)$-plane. We have thus completed the proof of the following proposition.

THEOREM 2. If the mean curvature $K_{m}$ is the same along all the points of any one of a given isothermal system of parallel plane sections, then the surface $\Sigma$ is either minimal, or it is a surface of revolution with axis perpendicular to the parallel plane sections, or it is a cylinder with elements parallel to the parallel plane sections.

For the discussion in the remainder of our paper, it will be found convenient to introduce the quantity $\Delta$ defined by the expression

$$
\Delta=\frac{\left(1+f_{y}^{2}\right) f_{x x}-2 f_{x} f_{y} f_{x y}+\left(1+f_{x}^{2}\right) f_{y y}}{1+f_{x}^{2}+f_{y}^{2}} .
$$

The mean curvature $K_{m}$ of the surface $\Sigma$ is given by the formula $K_{m}=\Delta \cos \gamma$, where $\gamma$ represents the angle between the normal to $\Sigma$ and the $z$-axis. Since $K_{m}$ and $\gamma$ are invariant under any motion in the $(x, y)$-plane, it is found that $\Delta$ remains unchanged under any motion in the $(x, y)$-plane.

For the surface $\Sigma$ defined by the Monge equation $z=f(x, y)$, it is found from (1), (3), and (12) that the system of parallel plane sections $x=$ const. is isothermal if and only if $\Delta f_{x} /\left(1+f_{y}^{2}\right)=\phi(x)$; or the system of parallel plane sections $y=$ const. is isothermal if and only if $\Delta f_{v} /\left(1+f_{x}^{2}\right)=\phi(y)$; or the system of parallel plane sections $z=$ const. is isothermal if and only if $\Delta /\left(f_{x}^{2}+f_{y}^{2}\right)=\phi(f)$. 
4. Surfaces for which every system of parallel plane sections is isothermal. As indicated in $\S 2$, if $\Sigma$ is any minimal surface (including the plane) or any sphere, then every system of parallel plane sections is isothermal. We shall show that this property is characteristic for the class of minimal surfaces and spheres. The following result is obtained.

THEOREM 3. If a surface $\Sigma$ possesses more than four distinct isothermal systems of parallel plane sections, all the planes of which are parallel to a given rectilinear direction $L$, then $\Sigma$ is a minimal surface or a sphere, and therefore every system of parallel plane sections is isothermal.

The proof will occupy the next few paragraphs.

Let us choose the given rectilinear direction $L$ as that of the $z$-axis. Then any system of parallel plane sections of the surface $\Sigma$ defined by the Monge equation $z=f(x, y)$, all of which are parallel to the given rectilinear direction $L$, is given by the equation $y=m x+$ const.

In the $(x, y)$-plane, let us apply the rotation

$$
\left(1+m^{2}\right)^{1 / 2} X=-m x+y, \quad\left(1+m^{2}\right)^{1 / 2} Y=x+m y .
$$

The system of parallel plane sections $y=m x+$ const. in the new coordinate system $(X, Y, z)$ is given by $X=$ const., and the surface $\Sigma$ : $z=f(x, y)$ is defined by the equation $z=f(X, Y)$.

Thus if $y=m x+$ const. is an isothermal system of parallel plane sections of $z=f(x, y)$, then $X=$ const. is an isothermal system of parallel plane sections of $z=f(X, Y)$. Therefore it follows by (1) that $\Delta f_{X} /\left(1+f_{Y}^{2}\right)$, where in the definition (12) of $\Delta$ the small letters $(x, y)$ are replaced by the capital letters $(X, Y)$, is a function of $X$ alone. That is, we must have

$$
f_{X} \Delta_{Y}=\Delta\left[-f_{X Y}+\frac{2 f_{X} f_{Y} f_{Y Y}}{1+f_{Y}^{2}}\right] .
$$

By means of (13), we can express the partial derivatives of $f$ and $\Delta$ with respect to $X$ and $Y$ in terms of those with respect to $x$ and $y$. Of course $\Delta$ remains unchanged under the rotation (13). Eliminating the capital letters $(X, Y)$ from (14) by means of (13), we obtain the result

$$
\begin{aligned}
& {\left[\left(1+f_{y}^{2}\right) m^{2}+2 f_{x} f_{y} m+\left(1+f_{x}^{2}\right)\right]\left[\left(\Delta f_{x y}+f_{x} \Delta_{y}\right) m^{2}\right.} \\
& \left.\quad-\left\{\left(f_{y} \Delta_{y}-f_{x} \Delta_{x}\right)+\Delta\left(f_{y y}-f_{x x}\right)\right\} m-\left(f_{y} \Delta_{x}+\Delta f_{x y}\right)\right] \\
& \quad-2 \Delta\left[f_{x} f_{y} m^{2}+\left(f_{x}^{2}-f_{y}^{2}\right) m-f_{x} f_{y}\right]\left[f_{y y} m^{2}+2 f_{x y} m+f_{x x}\right]=0 .
\end{aligned}
$$

This is an equation of the fourth degree in $m$. Therefore, for any surface $\Sigma$, corresponding to any rectilinear direction $L$, there are at most four distinct isothermal systems of parallel plane sections, all of which are parallel to $L$. 
Let a surface $\Sigma$ possess more than four such distinct isothermal systems, all parallel to the given rectilinear direction $L$. Choosing $L$ as the $z$-axis, it is seen that the preceding equation must be an identity in $m$. Hence the coefficients of the various powers of $m$ must be zero.

In the first place if $\Delta=0$, the equation (15) is identically zero, and the surface $\Sigma$ is a minimal surface. Henceforth we may assume $\Delta \neq 0$.

Upon setting the coefficient of $m^{4}$ and the term independent of $m$ equal to zero, we find

$$
f_{x} \Delta_{y}=\Delta\left[-f_{x y}+\frac{2 f_{x} f_{y} f_{y y}}{1+f_{y}^{2}}\right], \quad f_{y} \Delta_{x}=\Delta\left[-f_{x y}+\frac{2 f_{x} f_{y} f_{x x}}{1+f_{x}^{2}}\right]
$$

These two equations state that $x=$ const. and $y=$ const. are both isothermal systems of parallel plane sections.

Neither $f_{x}$ nor $f_{y}$ can be identically zero. Let us suppose that $f_{y}=0$. Since $\Delta \neq 0$, we find $f_{x x} \neq 0$. Also $\Delta_{y}=0$. Under these conditions, the identity (15) in $m$ leads to the conditions $f_{x} \Delta_{x}+\Delta f_{x x}=0, \Delta f_{x}^{2} f_{x x}=0$, the last of which is impossible. Hence $f_{y}$ can not be identically zero. A similar argument can be presented to show that $f_{x}$ can not be identically zero.

From the above discussion, it is seen that we need to consider only the case where $\Delta \neq 0, f_{x} \neq 0$, and $f_{y} \neq 0$. Also in the imaginary domain, it is seen that $f_{x}^{2} \neq-1$ and $f_{\nu}^{2} \neq-1$, for otherwise these would give imaginary minimal surfaces.

For the remainder of our proof, we shall find it convenient to introduce the quantities $S$ and $T$ defined by

$$
S=\frac{f_{x x}}{1+f_{x}^{2}}-\frac{f_{x y}}{f_{x} f_{y}}, \quad T=\frac{f_{y y}}{1+f_{y}^{2}}-\frac{f_{x y}}{f_{x} f_{y}} .
$$

We shall proceed to prove that, if $\Delta \neq 0$, the identity (15) will lead to the equations $S=T=0$, which are the partial differential equations defining any sphere in space.

Upon eliminating the partial derivatives of $\Delta$ from (15) by means of (16) and then setting the remaining coefficients of $m$ in (15) equal to zero, we obtain

$$
\begin{aligned}
\left(1+3 f_{x}^{2}+f_{y}^{2}+3 f_{x}^{2} f_{y}^{2}\right) S+\left(-1-2 f_{x}^{2}-2 f_{y}^{2}-f_{y}^{4}+2 f_{x}^{2} f_{y}^{2}\right) T & =0 \\
\left(1-2 f_{x}^{2}+f_{y}^{2}\right) S+\left(-1-f_{x}^{2}+2 f_{y}^{2}\right) T & =0 \\
\left(1+2 f_{x}^{2}+2 f_{y}^{2}+f_{x}^{4}-2 f_{x}^{2} f_{y}^{2}\right) S & \\
+\left(-1-f_{x}^{2}-3 f_{y}^{2}-3 f_{x}^{2} f_{y}^{2}\right) T & =0 .
\end{aligned}
$$

Multiplying the middle of the above equations by $-\left(1+f_{x}^{2}\right)$ and 
adding the result to the first of the preceding equations, we find

$$
\left(4 f_{x}^{2}+2 f_{x}^{4}+2 f_{x}^{2} f_{y}^{2}\right) S+\left(f_{x}^{4}-f_{y}^{4}-4 f_{y}^{2}\right) T=0 .
$$

Again multiplying the middle of equation (18) by $-\left(1+f_{y}^{2}\right)$ and adding the result to the last of equations (18), we obtain

$$
\left(f_{x}^{4}-f_{y}^{4}+4 f_{x}^{2}\right) S-\left(4 f_{y}^{2}+2 f_{x}^{2} f_{y}^{2}+2 f_{y}^{4}\right) T=0 .
$$

Subtracting (20) from (19), we discover that

$$
\left(f_{x}^{2}+f_{y}^{2}\right)^{2}(S-T)=0 .
$$

We shall prove that $S=T$. For otherwise we can get only the imaginary case of $f_{y}^{2}=-f_{x}^{2} \neq 0$. Substituting this into (18), we find

$$
\begin{aligned}
\left(1+2 f_{x}^{2}-3 f_{x}^{4}\right) S+\left(-1-3 f_{x}^{4}\right) T & =0, \\
\left(1-3 f_{x}^{2}\right) S+\left(-1-3 f_{x}^{2}\right) T & =0, \\
\left(1+3 f_{x}^{4}\right) S+\left(-1-2 f_{x}^{2}+3 f_{x}^{4}\right) T & =0 .
\end{aligned}
$$

Adding the first and third equations and dividing the result by 2 , we find $\left(1+f_{x}^{2}\right)(S-T)=0$. This is impossible since $f_{x}^{2} \neq-1$ and $S \neq T$. This proves that $S=T$.

Next we shall show that $S=T=0$. If that is not the case, the equations (18) lead us to the fact that $f_{y}=f_{x}=0$. Since this is impossible, it follows that $S=T=0$. Hence if the surface $\Sigma$ is not a minimal surface, its equation $z=f(x, y)$ must satisfy the simultaneous partial differential equations

$$
\frac{f_{x x}}{1+f_{x}^{2}}=\frac{f_{x y}}{f_{x} f_{y}}=\frac{f_{y y}}{1+f_{y}^{2}} .
$$

These are the partial differential equations of a sphere in general position. Thus the proof of Theorem 3 is complete.

5. A property of the sphere. The geodesics of a sphere are all great circles which are plane curves. We inquire if there are any other surfaces whose geodesics are all plane curves of nonzero curvature. In fact we prove the following precise result.

TheOREM 4. If a surface $\Sigma$ possesses more than $2 \infty^{1}$ plane nonrectilinear geodesics, then $\Sigma$ is a sphere.

The proof of this result is as follows. The $\infty^{2}$ geodesics of the surface $\Sigma$ defined by the Monge equation $z=f(x, y)$ are defined by the differential equation 


$$
\left(1+f_{x}^{2}+f_{y}^{2}\right) y^{\prime \prime}=\left(-f_{y}+y^{\prime} f_{x}\right)\left(f_{x x}+2 y^{\prime} f_{x y}+y^{\prime 2} f_{y y}\right),
$$

whereas the $\infty^{3}$ plane curves of $\Sigma$ are defined by the differential equation $^{3}$

$$
\begin{aligned}
& \left(f_{x x}+2 y^{\prime} f_{x y}+y^{\prime 2} f_{y y}\right) y^{\prime \prime \prime} \\
& \quad=\left(f_{x x x}+3 y^{\prime} f_{x x y}+3 y^{\prime 2} f_{x y y}+y^{\prime 3} f_{y y y}\right) y^{\prime \prime}+3\left(f_{x y}+y^{\prime} f_{y y}\right) y^{\prime \prime 2}
\end{aligned}
$$

Upon substituting (24) into (25), it is found that the geodesics which are plane curves must satisfy either the equation

$$
y^{\prime 2} f_{y y}+2 y^{\prime} f_{x y}+f_{x x}=0
$$

or the equation

$$
\begin{aligned}
y^{\prime 2}\left[\left(1+f_{y}^{2}\right) f_{x y}-f_{x} f_{y} f_{y y}\right]+y^{\prime}\left[\left(1+f_{y}^{2}\right) f_{x x}-\left(1+f_{x}^{2}\right) f_{y y}\right] \\
+\left[f_{x} f_{y} f_{x x}-\left(1+f_{x}^{2}\right) f_{x y}\right]=0 .
\end{aligned}
$$

Those geodesics which satisfy (26) must be straight lines. The surfaces which have exactly the $2 \infty^{1}$ straight lines defined by (26) are the ruled surfaces. If the roots of $y^{\prime}$ in (26) are equal, then only $\infty^{1}$ straight lines are in $\Sigma$ and $\Sigma$ is developable. Of course if $\Sigma$ has more than $2 \infty^{1}$ straight geodesics, it must be a plane.

If the geodesics are plane curves but not straight lines, they must satisfy the differential equation (27). Thus any surface $\Sigma$ has at most $2 \infty^{1}$ plane non-rectilinear geodesics.

If $\Sigma$ has more than $2 \infty^{1}$ such plane geodesics, then (27) must be an identity in $y^{\prime}$. Setting the various coefficient of $y^{\prime}$ equal to zero, we obtain the partial differential equations (23) for a sphere. This completes the proof of our Theorem $4 . .^{4}$

Columbia University AND

ILLINOIS INSTITUTE OF TECHNOLOGY

${ }^{3}$ Kasner, Dynamical trajectories and the $\infty^{3}$ plane sections of a surface, Proc. Nat. Acad. Sci. U.S.A. vol. 17 (1931) pp. 370-376.

4 Compare this Theorem 4 with the near-collineation problem. See Kasner, The characterization of collineations, Bull. Amer. Math. Soc. vol. 9 (1903) pp. 545-546. 